\title{
ON COMMUTING MATRICES AND EXPONENTIALS
}

\author{
CLÉMENT DE SEGUINS PAZZIS \\ (Communicated by Gail R. Letzter)
}

\begin{abstract}
Let $A$ and $B$ be matrices of $\mathrm{M}_{n}(\mathbb{C})$. We show that if $\exp (A)^{k} \exp (B)^{l}=\exp (k A+l B)$ for all integers $k$ and $l$, then $A B=B A$. We also show that if $\exp (A)^{k} \exp (B)=\exp (B) \exp (A)^{k}=\exp (k A+B)$ for every positive integer $k$, then the pair $(A, B)$ has property L of Motzkin and Taussky.

As a consequence, if $G$ is a subgroup of $\left(\mathrm{M}_{n}(\mathbb{C}),+\right)$ and $M \mapsto \exp (M)$ is a homomorphism from $G$ to $\left(\mathrm{GL}_{n}(\mathbb{C}), \times\right)$, then $G$ consists of commuting matrices. If $S$ is a subsemigroup of $\left(\mathrm{M}_{n}(\mathbb{C}),+\right)$ and $M \mapsto \exp (M)$ is a homomorphism from $S$ to $\left(\mathrm{GL}_{n}(\mathbb{C}), \times\right)$, then the linear subspace $\operatorname{Span}(S)$ of $\mathrm{M}_{n}(\mathbb{C})$ has property L of Motzkin and Taussky.
\end{abstract}

\section{INTRODUCTION}

\subsection{Notation and definition.}

i) We denote by $\mathbb{N}$ the set of non-negative integers.

ii) If $M \in \mathrm{M}_{n}(\mathbb{C})$, we denote by $e^{M}$ or $\exp (M)$ its exponential, by $\operatorname{Sp}(M)$ its set of eigenvalues.

iii) The $n \times n$ complex matrices $A, B$ are said to be simultaneously triangularizable if there exists an invertible matrix $P$ such that $P^{-1} A P$ and $P^{-1} B P$ are upper triangular.

iv) A pair $(A, B)$ of complex $n \times n$ matrices is said to have property $\mathrm{L}$ if for a special ordering $\left(\lambda_{i}\right)_{1 \leq i \leq n},\left(\mu_{i}\right)_{1 \leq i \leq n}$ of the eigenvalues of $A, B$, the eigenvalues of $x A+y B$ are $\left(x \lambda_{i}+y \mu_{i}\right)_{1 \leq i \leq n}$ for all values of the complex numbers $x, y$.

1.2. The problem. It is well-known that the exponential is not a group homomorphism from $\left(\mathrm{M}_{n}(\mathbb{C}),+\right)$ to $\left(\mathrm{GL}_{n}(\mathbb{C}), \times\right)$ if $n \geq 2$. Nevertheless, when $A$ and $B$ are commuting matrices of $\mathrm{M}_{n}(\mathbb{C})$, one has

$$
e^{A+B}=e^{A} e^{B}=e^{B} e^{A} .
$$

However (11) is not a sufficient condition for the commutativity of $A$ with $B$, nor even for $A$ and $B$ to be simultaneously triangularizable. Still, if

$$
\forall t \in \mathbb{R}, e^{t A} e^{t B}=e^{t B} e^{t A},
$$

or

$$
\forall t \in \mathbb{R}, e^{t(A+B)}=e^{t A} e^{t B}
$$

Received by the editors December 30, 2010 and, in revised form, May 24, 2011 and July 16, 2011.

2010 Mathematics Subject Classification. Primary 15A16; Secondary 15A22.

Key words and phrases. Matrix pencils, commuting exponentials, property L.

(C) 2012 American Mathematical Society Reverts to public domain 28 years from publication 
then a power series expansion at $t=0$ shows that $A B=B A$. In the 1950's, pairs of matrices $(A, B)$ of small size such that $e^{A+B}=e^{A} e^{B}$ have been under extensive scrutiny [3, 4, 6, 7, 9, 10. More recently, Wermuth [16, 17] and Schmoeger [14, 15] studied the problem of adding extra conditions on the matrices $A$ and $B$ for the commutativity of $e^{A}$ with $e^{B}$ to imply the commutativity of $A$ with $B$. A few years ago, Bourgeois (see [1]) investigated, for small $n$, the pairs $(A, B) \in \mathrm{M}_{n}(\mathbb{C})^{2}$ that satisfy

$$
\forall k \in \mathbb{N}, \quad e^{k A+B}=e^{k A} e^{B}=e^{B} e^{k A} .
$$

The main interest in this condition lies in the fact that, contrary to Conditions (2) and (3), it is not possible to use it to obtain information on $A$ and $B$ based only on the local behavior of the exponential around 0. Bourgeois showed that Condition (4) implies that $A$ and $B$ are simultaneously triangularizable if $n=2$, and produced a proof that this also holds when $n=3$. This last result is however false, as the following counterexample, communicated to us by Jean-Louis $\mathrm{Tu}$, shows: consider the matrices

$$
A_{1}:=2 i \pi\left[\begin{array}{lll}
1 & 0 & 0 \\
0 & 2 & 0 \\
0 & 0 & 0
\end{array}\right] \text { and } B_{1}:=2 i \pi\left[\begin{array}{ccc}
2 & 1 & 1 \\
1 & 3 & -2 \\
1 & 1 & 0
\end{array}\right] .
$$

Notice that $A_{1}$ and $B_{1}$ are not simultaneously triangularizable since they share no eigenvector (indeed, the eigenspaces of $A_{1}$ are the lines spanned by the three vectors of the canonical basis, and none of them is stabilized by $B_{1}$ ). However, for every $t \in \mathbb{C}$, a straightforward computation shows that the characteristic polynomial of $t A_{1}+B_{1}$ is

$$
X(X-2 i \pi(t+2))(X-2 i \pi(2 t+3)) .
$$

Then for every $t \in \mathbb{N}$, the matrix $t A_{1}+B_{1}$ has three distinct eigenvalues in $2 i \pi \mathbb{Z}$, hence is diagonalizable with $e^{t A_{1}+B_{1}}=I_{3}$. In particular $e^{B_{1}}=I_{3}$, and on the other hand, $e^{A_{1}}=I_{3}$. This shows that Condition (4) holds.

It then appears that one should strengthen Bourgeois' condition as follows in order to obtain at least the simultaneous triangularizability of $A$ and $B$ :

$$
\forall(k, l) \in \mathbb{Z}^{2}, \quad e^{k A+l B}=e^{k A} e^{l B} .
$$

Notice immediately that this condition implies that $e^{A}$ and $e^{B}$ commute. Indeed, if Condition (5) holds, then

$$
e^{B} e^{A}=\left(e^{-A} e^{-B}\right)^{-1}=\left(e^{-A-B}\right)^{-1}=e^{A+B}=e^{A} e^{B} .
$$

Therefore Condition (5) is equivalent to

$$
\forall(k, l) \in \mathbb{Z}^{2}, \quad e^{k A+l B}=e^{k A} e^{l B}=e^{l B} e^{k A} .
$$

Here is our main result.

Theorem 1. Let $(A, B) \in \mathrm{M}_{n}(\mathbb{C})^{2}$ be such that for all $(k, l) \in \mathbb{Z}^{2}$, $e^{k A+l B}=$ $e^{k A} e^{l B}$. Then $A B=B A$.

The following corollary is straightforward.

Theorem 2. Let $G$ be a subgroup of $\left(\mathrm{M}_{n}(\mathbb{C}),+\right)$ and assume that $M \mapsto \exp (M)$ is a homomorphism from $(G,+)$ to $\left(\mathrm{GL}_{n}(\mathbb{C}), \times\right)$. Then, for all $(A, B) \in G^{2}, A B=B A$. 
The key to the proof of Theorem 1 is

Proposition 3. Let $(A, B) \in \mathrm{M}_{n}(\mathbb{C})^{2}$. Assume that, for every $(k, l) \in \mathbb{Z}^{2}$, the matrix $k A+l B$ is diagonalizable and $\operatorname{Sp}(k A+l B) \subset \mathbb{Z}$. Then $A B=B A$.

For subsemigroups of $\left(\mathrm{M}_{n}(\mathbb{C}),+\right)$, Theorem 2 surely fails. A very simple counterexample is indeed given by the semigroup generated by

$$
A:=\left[\begin{array}{cc}
0 & 0 \\
0 & 2 i \pi
\end{array}\right] \text { and } B:=\left[\begin{array}{cc}
0 & 1 \\
0 & 2 i \pi
\end{array}\right] .
$$

One may however wonder whether a subsemigroup $S$ on which the exponential is a homomorphism must be simultaneously triangularizable. Obviously the additive semigroup generated by the matrices $A_{1}$ and $B_{1}$ above is a counterexample. Nevertheless, we will prove a weaker result, which rectifies and generalizes Bourgeois' results [1].

Proposition 4. Let $(A, B) \in \mathrm{M}_{n}(\mathbb{C})^{2}$ be such that $\forall k \in \mathbb{N}, e^{k A+B}=e^{k A} e^{B}=$ $e^{B} e^{k A}$. Then $(A, B)$ has property $L$.

Note that the converse is obviously false.

The proofs of Theorem 1 and of Proposition 4 have largely similar parts, so they will be tackled simultaneously. There are three main steps.

- We will prove Proposition 4 in the special case where $\operatorname{Sp}(A) \subset 2 i \pi \mathbb{Z}$ and $\operatorname{Sp}(B) \subset 2 i \pi \mathbb{Z}$. This will involve a study of the matrix pencil $z \mapsto A+z B$. We will then easily derive Proposition 3 using a refinement of the MotzkinTaussky theorem.

- We will handle the more general case $\operatorname{Sp}(A) \subset 2 i \pi \mathbb{Z}$ and $\operatorname{Sp}(B) \subset 2 i \pi \mathbb{Z}$ in Theorem 1 by using the Jordan-Chevalley decompositions of $A$ and $B$ together with Proposition 3 .

- In the general case, we will use an induction to reduce the situation to the previous one, both for Theorem 1 and Proposition 4 .

In the last section, we will prove a sort a generalized version of Proposition 4 for additive semigroups of matrices (see Theorem 11).

\section{AdDitive groups AND SEMigroups of MATRICES WITH AN INTEGRAL SPECTRUM}

\subsection{Notation.}

i) We denote by $\Sigma_{n}$ the group of permutations of $\{1, \ldots, n\}$, make it act on $\mathbb{C}^{n}$ by $\sigma \cdot\left(z_{1}, \ldots, z_{n}\right):=\left(z_{\sigma(1)}, \ldots, z_{\sigma(n)}\right)$, and consider the quotient set $\mathbb{C}^{n} / \Sigma_{n}$. The class of a list $\left(z_{1}, \ldots, z_{n}\right) \in \mathbb{C}^{n}$ in $\mathbb{C}^{n} / \Sigma_{n}$ will be denoted by $\left[z_{1}, \ldots, z_{n}\right]$.

ii) For $M \in \mathrm{M}_{n}(\mathbb{C})$, we denote by $\chi_{M}(X) \in \mathbb{C}[X]$ its characteristic polynomial, and we set

$$
\operatorname{OSp}(M):=\left[z_{1}, \ldots, z_{n}\right], \quad \text { where } \chi_{M}(X)=\prod_{k=1}^{n}\left(X-z_{k}\right) .
$$

iii) Given an integer $N \geq 1$, we set $\mathbb{U}_{N}(z):=\left\{\zeta \in \mathbb{C}: \zeta^{N}=z\right\}$. 


\subsection{Definition.}

Definition 1 (A reformulation of Motzkin-Taussky's Property L [1]).

A pair $(A, B) \in \mathrm{M}_{n}(\mathbb{C})^{2}$ has property $\mathrm{L}$ when there are $n$ linear forms $f_{1}, \ldots, f_{n}$ on $\mathbb{C}^{2}$ such that

$$
\forall(x, y) \in \mathbb{C}^{2}, \operatorname{OSp}(x A+y B)=\left[f_{k}(x, y)\right]_{1 \leq k \leq n} .
$$

Using the fact that the eigenvalues are continuous functions of the coefficients, it is obvious that a pair $(A, B) \in \mathrm{M}_{n}(\mathbb{C})^{2}$ has property $\mathrm{L}$ if and only if there are affine maps $f_{1}, \ldots, f_{n}$ from $\mathbb{C}$ to $\mathbb{C}$ such that

$$
\forall z \in \mathbb{C}, \operatorname{OSp}(A+z B)=\left[f_{k}(z)\right]_{1 \leq k \leq n} .
$$

2.3. Property $\mathbf{L}$ for pairs of matrices with an integral spectrum. We denote by $\mathcal{K}(\mathbb{C})$ the quotient field of the integral domain $H(\mathbb{C})$ of entire functions (i.e. analytic functions from $\mathbb{C}$ to $\mathbb{C})$. Considering id $\operatorname{id}_{\mathbb{C}}$ as an element of $\mathcal{K}(\mathbb{C})$, we may view $A+\operatorname{id}_{\mathbb{C}} B$ as a matrix of $\mathrm{M}_{n}(\mathcal{K}(\mathbb{C}))$. We define the generic number $p$ of eigenvalues of the pencil $z \mapsto A+z B$ as the number of the distinct eigenvalues of $A+\operatorname{id}_{\mathbb{C}} B$ in an algebraic closure of $\mathcal{K}(\mathbb{C})$. A complex number $z$ is called regular when $A+z B$ has exactly $p$ distinct eigenvalues, and exceptional otherwise. In a neighborhood of 0 , the spectrum of $A+z B$ may be classically described with Puiseux series as follows (see [2, chapter 7]): there exists a radius $r>0$, an integer $q \in\{1, \ldots, n\}$, positive integers $d_{1}, \ldots, d_{q}$ such that $n=d_{1}+\cdots+d_{q}$, and analytic functions $f_{1}, \ldots, f_{q}$ defined on a neighborhood of 0 such that

$$
\forall z \in \mathbb{C} \backslash\{0\}, \quad|z|<r \Rightarrow \chi_{A+z B}(X)=\prod_{k=1}^{q} \prod_{\zeta \in \mathbb{U}_{d_{k}}(z)}\left(X-f_{k}(\zeta)\right) .
$$

We may now prove the following result.

Proposition 5. Let $(A, B) \in \mathrm{M}_{n}(\mathbb{C})^{2}$. Assume that $\operatorname{Sp}(k A+B) \subset \mathbb{Z}$ for every $k \in \mathbb{N}$. Then $(A, B)$ has property $L$.

Proof. With the above notation, we prove that $f_{1}, \ldots, f_{q}$ are polynomial functions. For instance, consider $f_{1}$ and its power series expansion

$$
f_{1}(z)=\sum_{j=0}^{+\infty} a_{j} z^{j}
$$

Set $N:=d_{1}$ for convenience. Let $k_{0}$ be a positive integer such that $\frac{1}{k_{0}}<r$. For every integer $k \geq k_{0}, k f_{1}\left(k^{-\frac{1}{N}}\right)$ is an eigenvalue of $k A+B$; hence it is an integer. Therefore one has: for every integer $k \geq k_{0}$,

$$
(k+1) f_{1}\left((k+1)^{-\frac{1}{N}}\right)-k f_{1}\left(k^{-\frac{1}{N}}\right) \in \mathbb{Z} .
$$

For every integer $k \geq k_{0}$, the following equality holds:

$$
(k+1) f_{1}\left((k+1)^{-\frac{1}{N}}\right)-k f_{1}\left(k^{-\frac{1}{N}}\right)=a_{0}+\sum_{j \in \mathbb{N} \backslash\{0, N\}} a_{j}\left((k+1)^{1-\frac{j}{N}}-k^{1-\frac{j}{N}}\right) .
$$

Assume that $a_{j} \neq 0$ for some $j \geq 1$ with $j \neq N$, and define $s$ as the smallest such $j$. On the one hand, one has for every integer $j \in \mathbb{N}$,

$$
(k+1)^{1-\frac{j}{N}}-k^{1-\frac{j}{N}}=k^{1-\frac{j}{N}}\left(\left(1+\frac{1}{k}\right)^{1-\frac{j}{N}}-1\right) \underset{k \rightarrow+\infty}{\sim} k^{1-\frac{j}{N}} \frac{1-\frac{j}{N}}{k}=\left(1-\frac{j}{N}\right) k^{-\frac{j}{N}} .
$$


On the other hand, when $k \rightarrow+\infty$, one has

$$
\begin{aligned}
\sum_{j=s+N+1}^{+\infty} a_{j} k^{1-\frac{j}{N}} & =o\left(k^{-\frac{s}{N}}\right) \text { and } \\
\sum_{j=s+N+1}^{+\infty} a_{j}(k+1)^{1-\frac{j}{N}} & =o\left(k^{-\frac{s}{N}}\right) .
\end{aligned}
$$

It follows that

$$
\sum_{j \in \mathbb{N} \backslash\{0, N\}} a_{j}\left((k+1)^{1-\frac{j}{N}}-k^{1-\frac{j}{N}}\right) \underset{k \rightarrow+\infty}{\sim} a_{s}\left(1-\frac{s}{N}\right) k^{-\frac{s}{N}} .
$$

The sequence $\left((k+1) f_{1}\left((k+1)^{-\frac{1}{N}}\right)-k f_{1}\left(k^{-\frac{1}{N}}\right)-a_{0}\right)_{k \geq k_{0}}$ is discrete, converges to 0 and is not ultimately zero. This is a contradiction. Therefore $\forall j \in \mathbb{N} \backslash\{0, N\}, a_{j}=$ 0 . In the same way, one shows that, for every $k \in\{1, \ldots, q\}$, there exists a $b_{k} \in \mathbb{C}$ such that $f_{k}(z)=f_{k}(0)+b_{k} z^{d_{k}}$ in a neighborhood of 0 . It follows that, in a neighborhood of 0 ,

$$
\chi_{A+z B}(X)=\prod_{k=1}^{q}\left(X-f_{k}(0)-b_{k} z\right)^{d_{k}} .
$$

Therefore we found affine maps $g_{1}, \ldots, g_{n}$ from $\mathbb{C}$ to $\mathbb{C}$ such that, in a neighborhood of 0 ,

$$
\chi_{A+z B}(X)=\prod_{k=1}^{n}\left(X-g_{k}(z)\right) .
$$

The coefficients of these polynomials are polynomial functions of $z$ that coincide on a neighborhood of 0 ; therefore

$$
\forall z \in \mathbb{C}, \chi_{A+z B}(X)=\prod_{k=1}^{n}\left(X-g_{k}(z)\right) .
$$

The pair $(A, B)$ has property $\mathrm{L}$, and Proposition 5 is proven.

2.4. Commutativity for subgroups of diagonalizable matrices with an integral spectrum. Given a matrix $M \in \mathrm{M}_{n}(\mathbb{C})$ and an eigenvalue $\lambda$ of it, recall that the eigenprojection of $M$ associated to $\lambda$ is the projection onto $\operatorname{Ker}\left(M-\lambda I_{n}\right)^{n}$ alongside $\operatorname{im}\left(M-\lambda I_{n}\right)^{n}=\sum_{\mu \in \operatorname{Sp}(M), \mu \neq \lambda} \operatorname{Ker}\left(M-\mu I_{n}\right)^{n}$.

Here, we derive Proposition 3 from Proposition 5 . We start by explaining how Kato's proof [8, p. 85, Theorem 2.6] of the Motzkin-Taussky theorem [12] leads to the following refinement.

Theorem 6 (Refined Motzkin-Taussky theorem). Let $(A, B) \in \mathrm{M}_{n}(\mathbb{C})^{2}$ be a pair of matrices which satisfies property L. Assume that $B$ is diagonalizable and that $A+z_{0} B$ is diagonalizable for every exceptional point $z_{0}$ of the matrix pencil $z \mapsto$ $A+z B$. Then $A B=B A$.

Proof. We refer to the line of reasoning of [8, p. 85, Theorem 2.6] and explain how it may be adapted to prove Theorem 6. Denote by $p$ the generic number of eigenvalues of $z \mapsto A+z B$, and by $f_{1}, \ldots, f_{p}$ the $p$ distinct affine maps such that 
$\forall z \in \mathbb{C}, \operatorname{Sp}(A+z B)=\left\{f_{1}(z), \ldots, f_{p}(z)\right\}$. Denote by $\Omega$ the (open) set of regular points of $z \mapsto A+z B$, i.e.

$$
\Omega=\mathbb{C} \backslash\left\{z \in \mathbb{C}: \exists(i, j) \in\{1, \ldots, p\}^{2}: i \neq j \text { and } f_{i}(z)=f_{j}(z)\right\} .
$$

For $z \in \Omega$ and $i \in\{1, \ldots, p\}$, denote by $\Pi_{i}(z)$ the eigenprojection of $A+z B$ associated to the eigenvalue $f_{i}(z)$. Then $z \mapsto \Pi_{i}(z)$ is holomorphic on $\Omega$ for any $i \in\{1, \ldots, p\}$ (see [8, II.1.4]). Let $z_{0} \in \mathbb{C} \backslash \Omega$. Then $A+z_{0} B$ is diagonalizable and hence [8, p. 82, Theorem 2.3] shows that $z_{0}$ is a regular point for each map $z \mapsto \Pi_{i}(z)$. We deduce that the functions $\left(\Pi_{i}\right)_{i \leq p}$ are restrictions of entire functions. Since $B$ is diagonalizable, these functions are bounded at infinity (see the last paragraph of [8, p. 85]) and Liouville's theorem yields that they are constant. By a classical continuity argument (see [8, II.1.4, formula (1.16)]), we deduce that each eigenprojection of $B$ is a sum of some projections chosen among the $\left(\Pi_{i}(0)\right)_{i<p}$. As $B$ is diagonalizable, it is a linear combination of the $\left(\Pi_{i}(0)\right)_{i \leq p}$, which all commute with $A+z B$ for any regular $z$. Therefore $A B=B A$.

We now turn to the proof of Proposition 3.

Proof of Proposition 3 , Let $(A, B) \in \mathrm{M}_{n}(\mathbb{C})^{2}$. Assume that, for every $(k, l) \in \mathbb{Z}^{2}$, the matrix $k A+l B$ is diagonalizable and $\operatorname{Sp}(k A+l B) \subset \mathbb{Z}$. Proposition 5 then shows that $(A, B)$ has property L. For $k \in\{1, \ldots, n\}$, choose $f_{k}:(y, z) \mapsto \alpha_{k} y+\beta_{k} z$ such that

$$
\forall(y, z) \in \mathbb{C}^{2}, \operatorname{OSp}(y A+z B)=\left[f_{k}(y, z)\right]_{1 \leq k \leq n} .
$$

Since $\operatorname{Sp}(A)=\left\{\alpha_{1}, \ldots, \alpha_{n}\right\}$ and $\operatorname{Sp}(B)=\left\{\beta_{1}, \ldots, \beta_{n}\right\}$, the families $\left(\alpha_{k}\right)_{k \leq n}$ and $\left(\beta_{k}\right)_{k \leq n}$ are made of integers. It follows that the exceptional points of the matrix pencil $z \mapsto A+z B$ are rational numbers. As the matrix $A+\frac{l}{k} B=\frac{1}{k}(k A+l B)$ is diagonalizable for every $(k, l) \in(\mathbb{Z} \backslash\{0\}) \times \mathbb{Z}$, the refined Motzkin-Taussky theorem implies that $A B=B A$.

We now deduce the following special case of Theorem 1 .

Lemma 7. Let $(A, B) \in \mathrm{M}_{n}(\mathbb{C})^{2}$ be such that for all $(k, l) \in \mathbb{Z}^{2}, e^{k A+l B}=I_{n}$. Then $A B=B A$.

Proof. Recall that the solutions of the equation $e^{M}=I_{n}$ are the diagonalizable matrices $M$ such that $\operatorname{Sp}(M) \subset 2 i \pi \mathbb{Z}$ (see [5, Theorem 1.27]). In particular, for every $(k, l) \in \mathbb{Z}^{2}$, the matrix $k A+l B$ is diagonalizable and $\operatorname{Sp}(k A+l B) \subset 2 i \pi \mathbb{Z}$. Setting $A^{\prime}:=\frac{1}{2 i \pi} A$ and $B^{\prime}:=\frac{1}{2 i \pi} B$, we deduce that $\left(A^{\prime}, B^{\prime}\right)$ satisfies the assumptions of Proposition 3. It follows that $A^{\prime} B^{\prime}=B^{\prime} A^{\prime}$, and hence $A B=B A$.

\section{The CASe $\operatorname{Sp}(A) \subset 2 i \pi \mathbb{Z}$ And $\operatorname{Sp}(B) \subset 2 i \pi \mathbb{Z}$ IN TheOREm 1}

Proposition 8. Let $(A, B) \in \mathrm{M}_{n}(\mathbb{C})^{2}$ be such that $\forall(k, l) \in \mathbb{Z}^{2}, e^{k A+l B}=e^{k A} e^{l B}$, $\operatorname{Sp}(A) \subset 2 i \pi \mathbb{Z}$ and $\operatorname{Sp}(B) \subset 2 i \pi \mathbb{Z}$. Then $A B=B A$.

Proof. We consider the Jordan-Chevalley decompositions $A=D+N$ and $B=D^{\prime}+$ $N^{\prime}$, where $D$ and $D^{\prime}$ are diagonalizable, $N$ and $N^{\prime}$ are nilpotent and $D N=N D$ and $D^{\prime} N^{\prime}=N^{\prime} D^{\prime}$. Clearly, for every integer $k, k A=k D+k N$ (resp. $k B=k D^{\prime}+k N^{\prime}$ ) is the Jordan-Chevalley decomposition of $k A$ (resp. of $k B$ ), and $\operatorname{Sp}(k D)=\operatorname{Sp}(k A)=$ $k \operatorname{Sp}(A) \subset 2 i \pi \mathbb{Z}\left(\right.$ resp. $\left.\operatorname{Sp}\left(k D^{\prime}\right)=\operatorname{Sp}(k B)=k \operatorname{Sp}(B) \subset 2 i \pi \mathbb{Z}\right)$. This shows that

$$
e^{k A}=e^{k N} \text { and } e^{k B}=e^{k N^{\prime}} .
$$


Condition (6) may be written as

$$
\forall(k, l) \in \mathbb{Z}^{2}, \quad e^{k A+l B}=e^{k N} e^{l N^{\prime}}=e^{l N^{\prime}} e^{k N} .
$$

Note in particular that $e^{N}$ and $e^{N^{\prime}}$ commute. Since $N$ is nilpotent, we have

$$
N=\sum_{k=1}^{n-1} \frac{(-1)^{k+1}}{k}\left(e^{N}-I_{n}\right)^{k} .
$$

That shows that $N$ is a polynomial in $e^{N}$. Similarly $N^{\prime}$ is a polynomial in $e^{N^{\prime}}$. Therefore,

$$
N N^{\prime}=N^{\prime} N
$$

The above condition yields

$$
\forall(k, l) \in \mathbb{Z}^{2}, e^{k A+l B}=e^{k N+l N^{\prime}} .
$$

For any $(k, l) \in \mathbb{Z}^{2}, k N+l N^{\prime}$ is nilpotent since $N$ and $N^{\prime}$ are commuting nilpotent matrices. Hence $k N+l N^{\prime}$ is a polynomial in $e^{k N+l N^{\prime}}$. Since $k A+l B$ commutes with $e^{k A+l B}$, it commutes with $k N+l N^{\prime}$. Therefore

$$
e^{k D+l D^{\prime}}=e^{k A+l B} e^{-k N-l N^{\prime}}=I_{n} .
$$

In particular, this yields that $k D+l D^{\prime}$ is diagonalizable with $\operatorname{Sp}\left(k D+l D^{\prime}\right) \subset 2 i \pi \mathbb{Z}$, and the Jordan-Chevalley decomposition of $k A+l B$ is $k A+l B=\left(k D+l D^{\prime}\right)+$ $\left(k N+l N^{\prime}\right)$ as $k N+l N^{\prime}$ commutes with $k A+l B$.

By Lemma 7 , the matrices $D$ and $D^{\prime}$ commute. In particular $\left(D, D^{\prime}\right)$ has property L, which yields affine maps $f_{1}, \ldots, f_{n}$ from $\mathbb{C}$ to $\mathbb{C}$ such that

The set

$$
\forall z \in \mathbb{C}, \operatorname{OSp}\left(D+z D^{\prime}\right)=\left[f_{k}(z)\right]_{1 \leq k \leq n} .
$$

$$
E:=\left\{k \in \mathbb{Z}: \exists(i, j) \in\{1, \ldots, n\}^{2}: f_{i} \neq f_{j} \text { and } f_{i}(k)=f_{j}(k)\right\}
$$

is clearly finite. We may choose two distinct elements $a$ and $b$ in $\mathbb{Z} \backslash E$. The following equivalence holds:

$$
\forall(i, j) \in\{1, \ldots, n\}^{2}, f_{i}(a)=f_{j}(a) \Leftrightarrow f_{i}=f_{j} \Leftrightarrow f_{i}(b)=f_{j}(b) .
$$

Since $D$ and $D^{\prime}$ are simultaneously diagonalizable, it easily follows that $D+a D^{\prime}$ is a polynomial in $D+b D^{\prime}$ and conversely $D+b D^{\prime}$ is a polynomial in $D+a D^{\prime}$. Hence $N+a N^{\prime}$ and $N+b N^{\prime}$ both commute with $D+a D^{\prime}$ and $D+b D^{\prime}$. Since $N+a N^{\prime}$ and $N+b N^{\prime}$ commute with one another, we deduce that $A+a B=\left(D+a D^{\prime}\right)+\left(N+a N^{\prime}\right)$ commutes with $A+b B=\left(D+b D^{\prime}\right)+\left(N+b N^{\prime}\right)$. Since $a \neq b$, we conclude that $A B=B A$.

\section{Proofs of Theorem 1 and Proposition 4}

Definition 2. Let $(A, B) \in \mathrm{M}_{n}(\mathbb{C})^{2}$.

i) $(A, B)$ is said to be decomposable if there exists a non-trivial decomposition $\mathbb{C}^{n}=F \oplus G$ in which $F$ and $G$ are invariant linear subspaces for both $A$ and $B$.

ii) In the sequel, we consider, for $k \in \mathbb{N} \backslash\{0\}$, the function

$$
\gamma_{k}:(\lambda, \mu) \in \operatorname{Sp}\left(e^{A}\right) \times \operatorname{Sp}\left(e^{B}\right) \mapsto \lambda^{k} \mu \in \mathbb{C} .
$$

iii) For $\lambda \in \mathbb{C}$, we denote by $C_{\lambda}(M)$ the characteristic subspace of $M$ with respect to $\lambda$, i.e. $C_{\lambda}(M)=\operatorname{Ker}\left(M-\lambda I_{n}\right)^{n}$. 
Lemma 9. Assume that A satisfies Condition

$$
\forall(\lambda, \mu) \in \operatorname{Sp}(A)^{2}, \lambda-\mu \in 2 i \pi \mathbb{Q} \Rightarrow \lambda-\mu \in 2 i \pi \mathbb{Z} .
$$

Then there exists $k \in \mathbb{N} \backslash\{0\}$ such that $\gamma_{k}$ is one-to-one.

Proof. Assume that for every $k \in \mathbb{N} \backslash\{0\}$, there are distinct pairs $(\lambda, \mu)$ and $\left(\lambda^{\prime}, \mu^{\prime}\right)$ in $\operatorname{Sp}\left(e^{A}\right) \times \operatorname{Sp}\left(e^{B}\right)$ such that $\lambda^{k} \mu=\left(\lambda^{\prime}\right)^{k} \mu^{\prime}$. Since $\operatorname{Sp}\left(e^{A}\right) \times \operatorname{Sp}\left(e^{B}\right)$ is finite and $\mathbb{N} \backslash\{0\}$ is infinite, we may then find distinct pairs $(\lambda, \mu)$ and $\left(\lambda^{\prime}, \mu^{\prime}\right)$ in $\operatorname{Sp}\left(e^{A}\right) \times \operatorname{Sp}\left(e^{B}\right)$ and distinct non-zero integers $a$ and $b$ such that

$$
\lambda^{a} \mu=\left(\lambda^{\prime}\right)^{a} \mu^{\prime} \quad \text { and } \quad \lambda^{b} \mu=\left(\lambda^{\prime}\right)^{b} \mu^{\prime} .
$$

All those eigenvalues are non-zero and $\left(\frac{\lambda}{\lambda^{\prime}}\right)^{a-b}=1$ with $a \neq b$. It follows that $\frac{\lambda}{\lambda^{\prime}}$ is a root of unity. However $\lambda=e^{\alpha}$ and $\lambda^{\prime}=e^{\beta}$ for some $(\alpha, \beta) \in \operatorname{Sp}(A)^{2}$, which shows that $(a-b)(\alpha-\beta) \in 2 i \pi \mathbb{Z}$. Condition (7) yields $\alpha-\beta \in 2 i \pi \mathbb{Z}$; hence $\lambda=\lambda^{\prime}$. It follows that $\mu=\mu^{\prime}$, in contradiction with $(\lambda, \mu) \neq\left(\lambda^{\prime}, \mu^{\prime}\right)$.

Lemma 10. Assume that $\gamma_{1}$ is one-to-one and that $(A, B)$ satisfies Equality (5) (resp. Equality (4)). Then the characteristic subspaces of $e^{A}$ and $e^{B}$ are stabilized by $A$ and $B$.

Proof. Notice that $A+B$ commutes with $e^{A+B}$, hence commutes with $e^{A} e^{B}$. It thus stabilizes the characteristic subspaces of $e^{A} e^{B}$. Let us show that

$$
\forall \mu \in \operatorname{Sp}\left(e^{B}\right), C_{\mu}\left(e^{B}\right)=\bigoplus_{\lambda \in \operatorname{Sp}\left(e^{A}\right)} C_{\lambda \mu}\left(e^{A} e^{B}\right) .
$$

- Since $e^{B}$ and $e^{A}$ commute, $e^{A}$ stabilizes the characteristic subspaces of $e^{B}$. Considering the characteristic subspaces of the endomorphism of $C_{\mu}\left(e^{B}\right)$ induced by $e^{A}$, we find

$$
\forall \mu \in \operatorname{Sp}\left(e^{B}\right), C_{\mu}\left(e^{B}\right)=\bigoplus_{\lambda \in \operatorname{Sp}\left(e^{A}\right)}\left[C_{\lambda}\left(e^{A}\right) \cap C_{\mu}\left(e^{B}\right)\right] .
$$

- Let $(\lambda, \mu) \in \operatorname{Sp}\left(e^{A}\right) \times \operatorname{Sp}\left(e^{B}\right)$. Since $e^{A}$ and $e^{B}$ commute, they both stabilize $C_{\lambda}\left(e^{A}\right) \cap C_{\mu}\left(e^{B}\right)$ and induce simultaneously triangularizable endomorphisms of $C_{\lambda}\left(e^{A}\right) \cap C_{\mu}\left(e^{B}\right)$ each with a sole eigenvalue, respectively $\lambda$ and $\mu$ : it follows that

$$
C_{\lambda}\left(e^{A}\right) \cap C_{\mu}\left(e^{B}\right) \subset C_{\lambda \mu}\left(e^{A} e^{B}\right) .
$$

- Finally, the application $(\lambda, \mu) \mapsto \lambda \mu$ is one-to-one on $\operatorname{Sp}\left(e^{A}\right) \times \operatorname{Sp}\left(e^{B}\right)$. Therefore

$$
C_{\lambda \mu}\left(e^{A} e^{B}\right) \cap C_{\lambda^{\prime} \mu^{\prime}}\left(e^{A} e^{B}\right)=\{0\}
$$

for all distinct pairs $(\lambda, \mu)$ and $\left(\lambda^{\prime}, \mu^{\prime}\right)$ in $\operatorname{Sp}\left(e^{A}\right) \times \operatorname{Sp}\left(e^{B}\right)$.

One has

$$
\mathbb{C}^{n}=\bigoplus_{\mu \in \operatorname{Sp}\left(e^{B}\right)} C_{\mu}\left(e^{B}\right)=\bigoplus_{\mu \in \operatorname{Sp}\left(e^{B}\right)} \bigoplus_{\lambda \in \operatorname{Sp}\left(e^{A}\right)}\left[C_{\lambda}\left(e^{A}\right) \cap C_{\mu}\left(e^{B}\right)\right]
$$

and $\mathbb{C}^{n}$ is the sum of all the characteristic subspaces of $e^{A} e^{B}$. We deduce that

$$
\forall(\lambda, \mu) \in \operatorname{Sp}\left(e^{A}\right) \times \operatorname{Sp}\left(e^{B}\right), C_{\lambda \mu}\left(e^{A} e^{B}\right)=C_{\lambda}\left(e^{A}\right) \cap C_{\mu}\left(e^{B}\right) .
$$

This gives Equality (8).

We deduce that $A+B$ stabilizes every characteristic subspace of $e^{B}$. However this is also true of $B$ since it commutes with $e^{B}$. Hence both $A$ and $B$ stabilize the characteristic subspaces of $e^{B}$. Symmetrically, every characteristic subspace of $e^{A}$ is stabilized by both $A$ and $B$. 
Proof of Theorem 1 and Proposition 4. We use an induction on $n$. Both Theorem 1 and Proposition 4 obviously hold for $n=1$, so we fix $n \geq 2$ and assume that they hold for any pair $(A, B) \in \mathrm{M}_{k}(\mathbb{C})^{2}$ with $k \in\{1, \ldots, n-1\}$. Let $(A, B) \in \mathrm{M}_{n}(\mathbb{C})^{2}$ satisfy Equality (5) (resp. Equality (40)). Assume first that $(A, B)$ is decomposable. Then there exists $p \in\{1, \ldots, n-1\}$, a non-singular matrix $P \in \mathrm{GL}_{n}(\mathbb{C})$ and square matrices $A_{1}, B_{1}, A_{2}, B_{2}$ respectively in $\mathrm{M}_{p}(\mathbb{C})$, in $\mathrm{M}_{p}(\mathbb{C})$, in $\mathrm{M}_{n-p}(\mathbb{C})$ and in $\mathrm{M}_{n-p}(\mathbb{C})$ such that

$$
A=P\left[\begin{array}{cc}
A_{1} & 0 \\
0 & A_{2}
\end{array}\right] P^{-1} \text { and } B=P\left[\begin{array}{cc}
B_{1} & 0 \\
0 & B_{2}
\end{array}\right] P^{-1}
$$

Since the pair $(A, B)$ satisfies Equality (5) (resp. Equality (4)), it easily follows that this is also the case of $\left(A_{1}, B_{1}\right)$ and $\left(A_{2}, B_{2}\right)$; hence the induction hypothesis yields that $\left(A_{1}, B_{1}\right)$ and $\left(A_{2}, B_{2}\right)$ are commuting pairs (resp. have property L). Therefore $(A, B)$ is also a commuting pair (resp. has property $\mathrm{L}$ ).

From that point on, we assume that $(A, B)$ is indecomposable. We may also assume that $A$ satisfies Condition (7). Indeed, consider in general the finite set

$$
\mathcal{E}:=\mathbb{Q} \cap \frac{1}{2 i \pi}\left\{\lambda-\mu \mid(\lambda, \mu) \in \operatorname{Sp}(A)^{2}\right\}
$$

Since its elements are rational numbers, we may find some integer $p>0$ such that $p \mathcal{E} \subset \mathbb{Z}$. Replacing $A$ with $p A$, we notice that $(p A, B)$ still satisfies Equality (5) (resp. Equality (4)) and that it is a commuting pair (resp. satisfies property L) if and only if $(A, B)$ is a commuting pair (resp. satisfies property $\mathrm{L}$ ).

Assume now that $A$ satisfies Condition (7) as well as all the previous assumptions; i.e. $(A, B)$ is indecomposable and satisfies Equality (5) (resp. Equality (4)). By Lemma 9, we may choose $k \in \mathbb{N} \backslash\{0\}$ such that $\gamma_{k}$ is one-to-one. Replacing $A$ with $k A$, we lose no generality in assuming that $\gamma_{1}$ is one-to-one.

We can conclude: if $e^{B}$ has several eigenvalues, Lemma 10 contradicts the assumption that $(A, B)$ is indecomposable. It follows that $e^{B}$ has a sole eigenvalue, and for the same reason this is also true of $e^{A}$. Choosing $(\alpha, \beta) \in \mathbb{C}^{2}$ such that $\operatorname{Sp}\left(e^{A}\right)=\left\{e^{\alpha}\right\}$ and $\operatorname{Sp}\left(e^{B}\right)=\left\{e^{\beta}\right\}$, we find that $\exp \left(A-\alpha I_{n}\right)$ and $\exp \left(B-\beta I_{n}\right)$ both have 1 as their sole eigenvalue. We deduce that $\operatorname{Sp}\left(A-\alpha I_{n}\right) \subset 2 i \pi \mathbb{Z}$ and $\operatorname{Sp}\left(B-\beta I_{n}\right) \subset 2 i \pi \mathbb{Z}$. Set $A^{\prime}:=A-\alpha I_{n}$ and $B^{\prime}:=B-\beta I_{n}$. We now conclude the proofs of Theorem 1 and Proposition 4 by considering the two cases separately. - Case 1. $(A, B)$ satisfies Equality (5). The pair $\left(A^{\prime}, B^{\prime}\right)$ clearly satisfies Equality (5). Proposition 8 yields that $A^{\prime}$ commutes with $B^{\prime}$; hence $A B=B A$.

- Case 2. $(A, B)$ satisfies Equality (4). The pair $\left(A^{\prime}, B^{\prime}\right)$ obviously satisfies Equality (4). The matrices $e^{A^{\prime}}$ and $e^{B^{\prime}}$ commute and are therefore simultaneously triangularizable (see [13, Theorem 1.1.5]). Moreover, they have 1 as their sole eigenvalue. Therefore $e^{k A^{\prime}+B^{\prime}}=\left(e^{A^{\prime}}\right)^{k} e^{B^{\prime}}$ has 1 as sole eigenvalue for every $k \in \mathbb{N}$. Proposition 5 shows that $\left(\frac{1}{2 i \pi} A^{\prime}, \frac{1}{2 i \pi} B^{\prime}\right)$ has property L, which clearly entails that $(A, B)$ has property L.

Thus Theorem 1 and Proposition 4 are proven. 


\section{Additive SEMigroups ON Which the EXPONENTIAL} IS A HOMOMORPHISM

Notation 3 . We denote by $\mathbb{Q}_{+}$the set of non-negative rational numbers.

Definition 4. A linear subspace $V$ of $\mathrm{M}_{n}(\mathbb{C})$ has property $\mathrm{L}$ when there are $n$ linear forms $f_{1}, \ldots, f_{n}$ on $V$ such that

$$
\forall M \in V, \operatorname{OSp}(M)=\left[f_{k}(M)\right]_{1 \leq k \leq n} .
$$

In this short section, we prove the following result.

Theorem 11. Let $S$ be a subsemigroup of $\left(\mathrm{M}_{n}(\mathbb{C}),+\right)$ and assume that $M \mapsto$ $\exp (M)$ is a homomorphism from $(S,+)$ to $\left(\mathrm{GL}_{n}(\mathbb{C}), \times\right)$. Then $\operatorname{span}(S)$ has property $L$.

By Proposition 4, it suffices to establish the following lemma.

Lemma 12. Let $S$ be a subsemigroup of $\left(\mathrm{M}_{n}(\mathbb{C}),+\right)$. Assume that every pair $(A, B) \in S^{2}$ has property $L$. Then the linear subspace $\operatorname{span}(S)$ has property $L$.

Proof. Let $\left(A_{1}, \ldots, A_{r}\right)$ be a basis of $\operatorname{span}(S)$ formed of elements of $S$. For every $j \in\{1, \ldots, r\}$, we choose a list $\left(a_{1}^{(j)}, \ldots, a_{n}^{(j)}\right) \in \mathbb{C}^{n}$ such that

$$
\operatorname{OSp}\left(A_{j}\right)=\left[a_{k}^{(j)}\right]_{1 \leq k \leq n} .
$$

Since, for every $\left(p_{1}, \ldots, p_{r}\right) \in \mathbb{N}^{r}$, the pair $\left(\sum_{k=1}^{j-1} p_{k} A_{k}, A_{j}\right)$ has property L for every $j \in\{2, \ldots, r\}$, by induction we obtain a list $\left(\sigma_{1}, \ldots, \sigma_{r}\right) \in\left(\Sigma_{n}\right)^{r}$ such that

$$
\operatorname{OSp}\left(\sum_{j=1}^{r} p_{j} A_{j}\right)=\left[\sum_{j=1}^{r} p_{j} a_{\sigma_{j}(k)}^{(j)}\right]_{1 \leq k \leq n} .
$$

Multiplying by inverses of positive integers, we readily generalize this as follows: for every $\left(z_{1}, \ldots, z_{r}\right) \in\left(\mathbb{Q}_{+}\right)^{r}$, there exists a list $\left(\sigma_{1}, \ldots, \sigma_{r}\right) \in\left(\Sigma_{n}\right)^{r}$ such that

$$
\operatorname{OSp}\left(\sum_{j=1}^{r} z_{j} A_{j}\right)=\left[\sum_{j=1}^{r} z_{j} a_{\sigma_{j}(k)}^{(j)}\right]_{1 \leq k \leq n} .
$$

Now, we prove the following property, depending on $l \in\{0, \ldots, r\}$, by downward induction:

$$
\begin{gathered}
\mathcal{P}(l): \text { For every }\left(z_{1}, \ldots, z_{l}\right) \in \underset{\left(\mathbb{Q}_{+}\right)^{l}, \text { there exists a list }\left(\sigma_{1}, \ldots, \sigma_{r}\right) \in\left(\Sigma_{n}\right)^{r}}{ } \quad \text { satisfying } \\
\forall\left(z_{l+1}, \ldots, z_{r}\right) \in \mathbb{C}^{r-l}, \operatorname{OSp}\left(\sum_{j=1}^{r} z_{j} A_{j}\right)=\left[\sum_{j=1}^{r} z_{j} a_{\sigma_{j}(k)}^{(j)}\right]_{1 \leq k \leq n} .
\end{gathered}
$$

In particular, $\mathcal{P}(r)$ is precisely what we have just proven, whilst $\mathcal{P}(0)$ means that there exists a list $\left(\sigma_{1}, \ldots, \sigma_{r}\right) \in\left(\Sigma_{n}\right)^{r}$ such that, for every $\left(z_{1}, \ldots, z_{r}\right) \in \mathbb{C}^{r}$,

$$
\operatorname{OSp}\left(\sum_{j=1}^{r} z_{j} A_{j}\right)=\left[\sum_{j=1}^{r} z_{j} a_{\sigma_{j}(k)}^{(j)}\right]_{1 \leq k \leq n} .
$$

Therefore $\mathcal{P}(0)$ implies that $\operatorname{span}(S)$ has property L. 
Let $l \in\{1, \ldots, r\}$ be such that $\mathcal{P}(l)$ holds, and fix $\left(z_{1}, \ldots, z_{l-1}\right) \in\left(\mathbb{Q}_{+}\right)^{l-1}$. By $\mathcal{P}(l)$, for every $z_{l} \in \mathbb{Q}_{+}$, we may choose a list $\left(\sigma_{1}^{z_{l}}, \ldots, \sigma_{r}^{z_{l}}\right) \in\left(\Sigma_{n}\right)^{r}$ such that

$$
\forall\left(z_{l+1}, \ldots, z_{r}\right) \in \mathbb{C}^{r-l}, \operatorname{OSp}\left(\sum_{j=1}^{r} z_{j} A_{j}\right)=\left[\sum_{j=1}^{r} z_{j} a_{\sigma_{j}^{z_{l}(k)}}^{(j)}\right]_{1 \leq k \leq n} .
$$

Since $\left(\Sigma_{n}\right)^{r}$ is finite and $\mathbb{Q}_{+} \cap(0,1)$ is infinite, some list $\left(\sigma_{1}, \ldots, \sigma_{r}\right) \in\left(\Sigma_{n}\right)^{r}$ equals $\left(\sigma_{1}^{z_{l}}, \ldots, \sigma_{r}^{z_{l}}\right)$ for infinitely many values of $z_{l}$ in $\mathbb{Q}_{+} \cap(0,1)$. Fixing $\left(z_{l+1}, \ldots, z_{r}\right) \in$ $\mathbb{C}^{r-l}$, we deduce the identity

$$
\forall z_{l} \in \mathbb{C}, \quad \chi_{\sum_{j=1}^{r} z_{j} A_{j}}(X)=\prod_{k=1}^{n}\left(X-\sum_{j=1}^{r} z_{j} a_{\sigma_{j}(k)}^{(j)}\right)
$$

by remarking that, on both sides, the coefficients of the polynomials are polynomials in $z_{l}$. Hence

$$
\forall\left(z_{l}, \ldots, z_{r}\right) \in \mathbb{C}^{r-l+1}, \operatorname{OSp}\left(\sum_{j=1}^{r} z_{j} A_{j}\right)=\left[\sum_{j=1}^{r} z_{j} a_{\sigma_{j}(k)}^{(j)}\right]_{1 \leq k \leq n} .
$$

This proves that $\mathcal{P}(l-1)$ holds.

\section{ACKNOWLEDGEMENT}

The author would like to thank the referee for helping enhance the quality of this article in a very significant way.

\section{REFERENCES}

[1] G. Bourgeois, On commuting exponentials in low dimensions, Linear Algebra Appl. 423 (2007) 277-286. MR2312407 (2008d:39030)

[2] G. Fischer. Plane Algebraic Curves, Student Mathematical Library, Volume 15, AMS, 2001. MR.1836037 (2002g:14042)

[3] M. Fréchet, Les solutions non-commutables de l'équation matricielle $e^{x+y}=e^{x} e^{y}$, Rend. Circ. Math. Palermo 2 (1952) 11-27. MR0049857 (14:237a)

[4] M. Fréchet, Les solutions non-commutables de l'équation matricielle $e^{x+y}=e^{x} e^{y}$, Rectification, Rend. Circ. Math. Palermo 2 (1953) 71-72. MR0057836 (15:279h)

[5] N.J. Higham. Functions of Matrices. Theory and Computation, SIAM, 2008. MR2396439 (2009b:15001)

[6] C.W. Huff, On pairs of matrices (of order two) $A, B$ satisfying the condition $e^{A+B}=e^{A} e^{B} \neq$ $e^{B} e^{A}$, Rend. Circ. Math. Palermo 2 (1953) 326-330. MR0062706 (16:4c)

[7] A.G. Kakar, Non-commuting solutions of the matrix equation $\exp (X+Y)=\exp (X) \exp (Y)$, Rend. Circ. Math. Palermo 2 (1953) 331-345. MR0062708 (16:4e)

[8] T. Kato. Perturbation Theory for Linear Operators, Grundlehren der Mathematischen Wissenschaften, Second edition, Springer-Verlag, 1976. MR0407617 (53:11389)

[9] K. Morinaga, T. Nono, On the non-commutative solutions of the exponential equation $e^{x} e^{y}=$ $e^{x+y}$, J. Sci. Hiroshima Univ. (A)17 (1954) 345-358. MR0066337 (16:558f)

[10] K. Morinaga, T. Nono, On the non-commutative solutions of the exponential equation $e^{x} e^{y}=$ $e^{x+y}$ II, J. Sci. Hiroshima Univ. (A)18 (1954) 137-178. MR0072104 (17:228h)

[11] T.S. Motzkin, O. Taussky, Pairs of matrices with property L, Trans. Amer. Math. Soc. 73 (1952) 108-114. MR0049855(14:236e)

[12] T.S. Motzkin, O. Taussky, Pairs of matrices with property L (II), Trans. Amer. Math. Soc. 80 (1955) 387-401. MR0086781 (19:242c)

[13] H. Radjavi, P. Rosenthal, Simultaneous Triangularization, Universitext, Springer-Verlag (2000). MR1736065 (2001e:47001)

[14] C. Schmoeger, Remarks on commuting exponentials in Banach algebras, Proc. Amer. Math. Soc. 127 (5) (1999) 1337-1338. MR1476391 (99h:46090) 
[15] C. Schmoeger, Remarks on commuting exponentials in Banach algebras II, Proc. Amer. Math. Soc. 128 (11) (2000) 3405-3409. MR.1691002 (2001b:46077)

[16] E.M.E. Wermuth, Two remarks on matrix exponentials, Linear Algebra Appl. 117 (1989) 127-132. MR993038 (90e:15019)

[17] E.M.E. Wermuth, A remark on commuting operator exponentials, Proc. Amer. Math. Soc. 125 (6) (1997) 1685-1688. MR.1353407 (97g:39011)

Lycée Privé Sainte-Geneviève, 2, Rue de l’École des Postes, 78029 Versailles Cedex, FRANCE

E-mail address: dsp.prof@gmail.com 